\title{
Genetic diversity at major histocompatibility complex and its effect on production and immune traits in indigenous chicken breeds of India
}

\author{
Santosh Haunshi, Divya Devara, Kannaki Ramasamy, Rajkumar Ullengala, and Rudra Nath Chatterjee \\ ICAR-Directorate of Poultry Research, Rajendranagar, Hyderabad 500030, India
}

Correspondence: Santosh Haunshi (santosh.haunshi@icar.gov.in, santosh575g@gmail.com)

Received: 21 October 2019 - Revised: 9 April 2020 - Accepted: 12 May 2020 - Published: 16 June 2020

\begin{abstract}
The genetic diversity at major histocompatibility complex (MHC) in indigenous chicken breeds of India (Ghagus and Nicobari) in comparison with the White Leghorn (WLH) breed was investigated by genotyping the MHC-linked LEI0258 marker. Altogether 38 alleles and 96 genotypes were observed among three breeds. The observed and effective alleles were highest in Ghagus $(23,8.3)$ followed by Nicobari $(14,3.2)$ and WLH $(10$ and 2.2) breeds. The size of alleles ranged from 193 to $489 \mathrm{bp}$ in Ghagus, 193 to $552 \mathrm{bp}$ in Nicobari and 241 to $565 \mathrm{bp}$ in the WLH breed. The number of private alleles was also highest in Ghagus (18) followed by Nicobari (8) and WLH (5) breeds. The most frequent allele was $261 \mathrm{bp}$ in WLH (66\%), $343 \mathrm{bp}$ in Nicobari $(50.4 \%)$ and $309 \mathrm{bp}$ in the Ghagus $(28.15 \%)$ breed. Observed and expected heterozygosities were highest in Ghagus $(0.83$, $0.88)$ followed by Nicobari $(0.58,0.68)$ and WLH $(0.53,0.54)$. The genetic distance (Nei) between Ghagus and Nicobari breeds (2.24) was higher as compared to that of Ghagus and WLH (1.23) and that between Nicobari and WLH breeds (0.89). Association analysis revealed significant influence of MHC alleles on body weight, egg production in Ghagus and WLH breeds and antibody titres to Newcastle disease vaccine in the Nicobari breed.
\end{abstract}

\section{Introduction}

Slow-growing indigenous chickens possess unique attributes like attractive multicoloured plumage, hardiness, an ability to adapt to low input suboptimal rearing conditions and survive under harsh environments, broodiness, perceived desirable taste and flavour of meat and eggs, etc. Rearing of indigenous chickens generates subsidiary income by utilizing minimum inputs and minimum human attention. It also helps in gender empowerment and social upliftment of the rural/tribal people as mostly women and children are involved in rearing of indigenous chickens besides providing household nutritional security. Further, they cater to the needs of consumers for coloured birds and light-brown-shelled eggs in the niche market. Indigenous chickens are reported to be resistant to bacterial, protozoal, fungal and parasitic diseases as they were subjected to many years of natural selection under free-range or backyard systems of rearing (Besbes, 2009). Previous studies have reported the differences in immune competence traits between indigenous and improved chicken breeds owing to different genetic backgrounds. Nicobari, a slow-growing indigenous breed was reported to be relatively resistant to viral diseases like Newcastle disease, Marek's disease (Rai and Ahlawat, 1995) and infectious bursal disease (Sunder et al., 2004; Chatterjee and Yadav, 2008). Furthermore, expression profiling of various pattern recognition receptor genes in indigenous and White Leghorn (WLH) birds revealed the highest expression of TLRILB, MDA5, LGP2, B-Lec, IL1 $\beta$ and $I L 8$ genes in Ghagus in contrast to WLH birds (Haunshi et al., 2017). These receptor genes are known to play a significant role in innate immune competence. In another study, it was demonstrated that expression of the TLR4 gene in lipopolysaccharide treated peripheral blood mononuclear cells was significantly higher in indigenous chicken breeds (Aseel and Ghagus) when compared to improved (Dahlem Red and Broilers) chickens (Karnati et al., 2015). Similarly, the expression of TLR4, TLR5 and TLR7 genes was significantly higher in indigenous breeds (Kadaknath and Aseel) than those of the WLH breed (Kannaki et al., 2010). General immune competence as assessed by cutaneous basophil hy- 
persensitivity response against PHA-P and cytotoxic T lymphocytes $(\mathrm{CD} 8+)$ count was also found to be higher in indigenous chicken breeds compared to the Dahlem Red breed (Yadav et al., 2018). Recently in our challenge study, it was observed that the Nicobari breed was relatively resistant to fowl typhoid (Pastorella multocida) infection as mortality of the Nicobari breed was remarkably lesser as compared to the improved Vanaraja cross (Kannaki Ramasamy et al., unpublished data). Therefore, it is evident that the genetic background of the chicken is known to influence the disease resistance and immune competence traits (Emam et al., 2014).

Major histocompatibility complex (MHC) in chickens is known to play a significant role in disease resistance, immune competence and production traits (Miller and Taylor, 2016) including resistance to ectoparasites like northern fowl mites (Owen et al., 2008). Associations between the haplotypes of the MHC-B locus and responses to Rous sarcoma virus (Suzuki et al., 2010) and resistance to avian coronavirus (Banat et al., 2013) were also demonstrated. MHC-linked LEI0258 alleles were reported to be significantly associated with production traits in WLH (Lunden et al., 1993) and indigenous Khorasan chickens of Iran (Nikbakht and Esmailnejad, 2015). Recently, it was shown that LEI0258 alleles were associated with humoral and cell mediated immune response in broiler chickens (Esmailnejad et al., 2017). Alleles of the LEI0258 VNTR marker are associated with the serologically defined MHC B haplotypes (Fulton et al., 2006). Various studies have used genotyping of the LEI0258 marker to assess the genetic diversity at MHC (Nikbakht et al., 2013; Han et al., 2013; Guangxin et al., 2014). In a study, Baelmans et al. (2005) detected completely unknown haplotypes using serological method in four lines of indigenous chickens of India, thus reflecting the potentially interesting genetic pool at MHC. Therefore, the present study was carried out with the objective of investigating the genetic variability at MHC in indigenous and WLH breeds of chicken and to study the association of alleles of LEI0258 on growth, production, immunity and survivability traits in indigenous chicken breeds in comparison with the improved WLH breed.

\section{Materials and methods}

\subsection{Experimental birds}

A total of 363 birds from three breeds of chicken were used for the present study. Ghagus and Nicobari were the indigenous breeds of India, while a control population of White Leghorn (WLH) was the improved chicken breed. Breeding stocks of all three breeds were kept at the institute farm. Ghagus is phenotypically characterized by brown to redcoloured plumage, pea comb, white skin and brown eyes with high incidence of broodiness behaviour. Nicobari is small bird with brownish matt-coloured plumage and short legs that originated in the Andaman and Nicobar islands of India (Ahlawat et al., 2004). All three breeds were maintained as random bred closed populations. Chicks from three breeds were hatched simultaneously and reared on a floor (deep litter) up to 20 weeks of age, then in individual cages from 21 to 72 weeks of age in an open-sided house. The details of rearing and management, healthcare and vaccinations of birds were described previously (Haunshi et al., 2019).

\subsection{Blood sampling and DNA isolation}

At 20 weeks of age, blood samples from female birds of 119 Ghagus, 125 Nicobari and 119 WLH were collected from a wing vein of birds in 0.5 M EDTA anticoagulant, thoroughly mixed and stored at $-20^{\circ} \mathrm{C}$ till further use. Genomic DNA from whole blood was extracted using a phenolchloroform-isoamyl alcohol method (Sambrook and Russell, 2001). Briefly, about $50 \mu \mathrm{L}$ of whole blood was mixed with lysis buffer and proteinase- $\mathrm{K}$ and vortexed. The mixture was incubated in a water bath at $37^{\circ} \mathrm{C}$ for $12 \mathrm{~h}$. Equal quantity of phenol was added to the incubated mixture, gently mixed and centrifuged at $12000 \mathrm{~g}$ for $10 \mathrm{~min}$. Supernatant was transferred to a fresh tube. Further extraction was done with phenol : chloroform : isoamyl alcohol $(25: 24: 1)$ and chloroform: isoamyl alcohol $(24: 1)$. Finally, aqueous phase was transferred to a fresh tube having $3 \mathrm{M}$ sodium acetate and two volumes of chilled isopropanol and mixed gently. The pelleted DNA was dissolved in nuclease-free water. Quality and quantity of DNA was checked with the NanoDrop spectrophotometer. The present experiment was approved by the Institutional Animal Ethics Committee (IEAC).

\subsection{Genotyping by PCR}

Alleles of the LEI0258 marker were identified through PCR. The nucleotide sequences of primers used for amplification of the LEI0258 marker were CACGCAGCAGAACTTGGTAAGG forward and AGCTGTGCTCAGTCCTCAGTGC reverse (McConnell et al., 1999). Forward primer to amplify the LEI0258 marker through PCR was labelled with 6-FAM dye. PCR was carried out using $0.13 \mu \mathrm{M}$ each for forward and reverse primers, $1.5 \mathrm{mM} \mathrm{MgCl}_{2}, 0.6$ units of Dream Taq DNA polymerase (Thermo Fisher Scientific) $200 \mu \mathrm{M}$ of dNTPs, and $50 \mathrm{ng}$ of template (genomic DNA) in a final reaction volume of $25 \mu \mathrm{L}$ in a thermal cycler (Veriti, Applied Biosystems, Foster City, USA). Amplification conditions used were initial denaturation at $95^{\circ} \mathrm{C}$ for $10 \mathrm{~min}$ followed by 40 cycles of $95^{\circ} \mathrm{C}$ for $20 \mathrm{~s}, 62^{\circ} \mathrm{C}$ for $20 \mathrm{~s}$ and $72^{\circ} \mathrm{C}$ for $20 \mathrm{~s}$, followed by a final extension of $72^{\circ} \mathrm{C}$ for $10 \mathrm{~min}$. Initially, PCR products were resolved on $3.0 \%$ agarose gel in $1 \mathrm{X}$ TBE buffer at $80 \mathrm{~V}$ for $1.5 \mathrm{~h}$ to determine the approximate size of alleles. Subsequently, the left-over PCR product was mixed with Hi-Di Formamide and LIZ-500 marker, denatured, and run on an ABI-3500 genetic analyser (Chromous Biotech, Bengaluru). Fragment analysis was performed using the GeneMapper software to determine the size of alleles of the LEI0258 marker precisely. 


\subsection{Sequencing}

The exact size of alleles (19 alleles which were predominantly observed among three breeds) of LEI0258 was determined by sequencing the PCR products obtained using primers that bind just outside of the LEI0258 marker. The primers used to acquire entire region of the LEI0258 marker were TCGGGAAAAGATCTGAGTCATTG forward (CAJF01F) and TGATTTTCAGATCGCGTTCCTC (CAJF01R) for the reverse direction (Fulton et al., 2006). The PCR was performed in a final volume of $25 \mu \mathrm{L}$ with $50 \mathrm{ng}$ of chicken genomic DNA, $2.5 \mu \mathrm{L}$ of buffer $(10 \times)$, $0.67 \mu \mathrm{L}$ of each forward and reverse primers, $0.5 \mu \mathrm{L}$ of $10 \mathrm{mM}$ dNTP, $0.6 \mathrm{U}$ of Taq DNA polymerase, and water. The PCR cycles included $95^{\circ} \mathrm{C}$ for $10 \mathrm{~min}, 40$ cycles of $95^{\circ} \mathrm{C}$ for $20 \mathrm{~s}, 62^{\circ} \mathrm{C}$ for $20 \mathrm{~s}, 72^{\circ} \mathrm{C}$ for $20 \mathrm{~s}$ and $72^{\circ} \mathrm{C}$ for $10 \mathrm{~min}$. The PCR products were separated on agarose gel, and desired fragments were gel eluted using a QIAquick gel extraction kit (Qiagen GmbH, Hilden, Germany) following manufacturer's instructions. These eluted products were sequenced in both directions using BigDye terminator sequencing on an ABI 3730 sequencer (Bioserve Biotechnologies Pvt. Ltd., Hyderabad, India). Sequences of both forward and reverse directions were aligned using BioEdit software to determine the consensus sequence of alleles. ClustalW functions of the BioEdit software were used to investigate the SNP and indel polymorphisms in the flanking regions of R12 and R13 repeats.

\subsection{Population genetic analysis}

The alleles were identified by their sizes. The observed $\left(N_{\mathrm{a}}\right)$ and effective $\left(N_{\mathrm{e}}\right)$ allele number, allele frequency, expected, unbiased expected, and observed heterozygosities ( $H_{\mathrm{e}}, u H_{\mathrm{e}}$ and $H_{\mathrm{o}}$, respectively) for the LEI0258 locus were estimated using GenAlEx software version 6.502 (Peakall and Smouse, 2006, 2012). The amount of gene diversity was measured by the number of alleles and the unbiased expected heterozygosity, according to the formula proposed by Nei (1973). Genetic identity and genetic distance among the three breeds were calculated from allele frequency according to Nei's unbiased estimates (1978). Deviation from HardyWeinberg equilibrium (HWE) was estimated using the analysis of molecular variance (AMOVA) function of GenAlEx software.

\subsection{Association analysis}

The data on growth traits such as body weight and shank length at $4,8,16$, and 40 weeks of age, production traits such as average age at first egg, egg production up to 72 weeks of age, immunity traits like antibody titres to Newcastle disease vaccine (NDV), and natural antibody (NAb) titres to rabbit $\mathrm{RBC}$ (RRBC) at 20 weeks of age recorded in the three breeds previously (Haunshi et al., 2019) were used for the association study. The association of LEI0258 alleles with various traits was determined using following regression model

$Y_{i}=\mu+\Sigma b_{j} f_{i j}+\varepsilon_{i}$,

where, $Y_{i}$ is a dependent variable for specific trait in $i$ th chicken; $\mu$ is a general mean; $f_{i j}$ is the copy number of the $j$ th allele of LEI0258 in the $i$ th chicken; $b_{j}$ is half the substitution effect of the $j$ th allele; and $\varepsilon_{i}$ is the residual effect (Esmailnejad et al., 2017). For each allele, all hens were considered as either carrier (1) or non-carrier (0), and then coefficient effect of each allele was determined in comparison to the reference allele. The most frequent allele in each breed was designated as the reference allele, and the association was carried out using regression analysis (SPSS Ver. 12).

\section{Results}

A total of 19 alleles (Table 1) were sequenced in bi-direction and aligned, and consensus size was determined. It was observed that the sizes of alleles detected by capillary electrophoresis (fragment lengths) and sequencing did not match. Overall the difference in size ranged from 1 to $20 \mathrm{bp}$. There was a pattern in the size difference. The smaller the size of alleles, the smaller the difference in size was. The size difference of $1 \mathrm{bp}$ was observed for 195, 262 and $296 \mathrm{bp}$ alleles. Likewise, the size differences were found to be 3 bp for 312 and $346 \mathrm{bp}$ alleles, $4 \mathrm{bp}$ for 361,373 and $385 \mathrm{bp}$ alleles, $6 \mathrm{bp}$ for 451 and $495 \mathrm{bp}$ alleles, $13 \mathrm{bp}$ for $552 \mathrm{bp}$ allele, and finally $20 \mathrm{bp}$ for 572 and $585 \mathrm{bp}$ alleles. This pattern of difference in size was used for extrapolating the size of remaining genotyped alleles (Fulton et al., 2006). Similar to the findings of the present study, a size difference of 2 to $17 \mathrm{bp}$ was reported by Mwambene et al. (2019). However, Han et al. (2013) reported a higher ( 1 to $65 \mathrm{bp}$ ) difference in the size of genotyped allele and that determined by the sequencing.

Previous studies have reported two types of polymorphisms in the LEI0258 marker. One is the number of repeats of the R13 and R12 motifs, and the other is the number of SNPs and indels upstream $(-78,-72$ or $-62 \mathrm{bp})$ and downstream $(88,67$ or $51 \mathrm{bp})$ of these conserved repeat motifs (Fulton et al., 2006; Guangxin et al., 2014; Chazara et al., 2013). The number of $13 \mathrm{bp}$ (CTATGTCTTCTTT) repeat (R13) varied from 1 to 29 times, while that of $12 \mathrm{bp}$ (CTTTCCTTCTTT) repeat (R12) varied from 3 to 27 times. We have observed one deletion (the TT indel at position -30 to $-29 \mathrm{bp}$, i.e. $29 \mathrm{bp}$ before the R13 repeats) and one SNP (G/A, -28) in an upstream position (77 bp). Two indels (ATTTTGAG at +11 to $+18 \mathrm{bp}$ and $/ \mathrm{A}$ at $+28 \mathrm{bp}$ ) and two SNPs $(\mathrm{A} / \mathrm{T},+26$ and A/T, +34) were identified downstream $(68 \mathrm{bp})$ of $\mathrm{R} 12$ repeats. The position of indel marker "ATTTTGAG" (+11 to +18 bp downstream of last R12 repeat) is different from that reported previously (Fulton et al., 2006; Han et al., 2013; Chazara et al., 2013). A conserved 8 bp sequence, "CTTTCCTT", was observed after the final $\mathrm{R} 12$ repeat in all the sequenced samples. BLASTting of all 


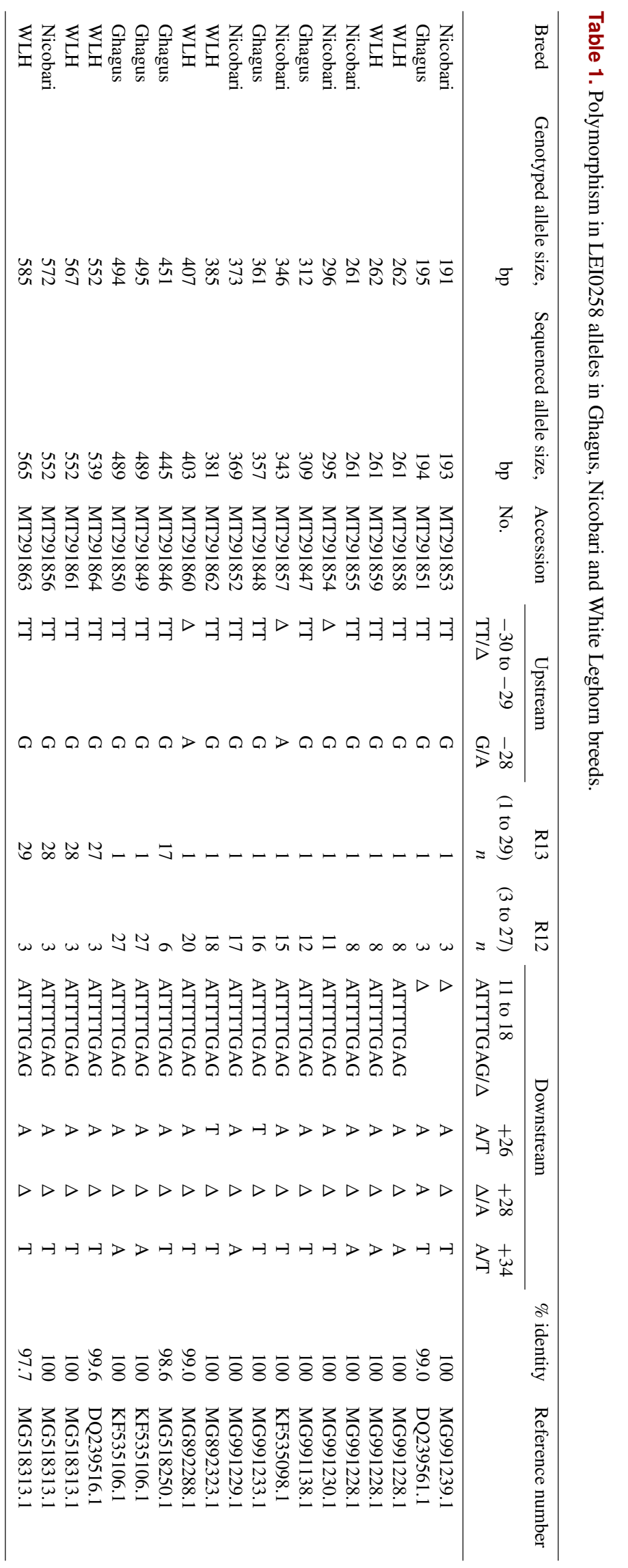


19 sequenced alleles in the NCBI nucleotide database indicated that 14 alleles are identical $(100 \%)$ to their corresponding accessions from the GenBank database. However, similarities of five sequences against their corresponding accessions in GenBank were slightly less than $100 \%$ (Table 1). All the 19 sequences submitted to NCBI GenBank were provided with accession numbers which ranged from MT291846 to MT291864.

Genetic diversity at major histocompatibility (MHC) in Ghagus, Nicobari and WLH breeds was studied by genotyping the MHC-linked LEI0258 marker. Overall 38 alleles with size ranging from 193 to 565 bp and 96 genotypes were observed in three breeds (Fig. 1). In Ghagus, a total of 23 alleles and 52 genotypes were found. The size of alleles ranged from 193 to $489 \mathrm{bp}$. The most frequent allele was $309 \mathrm{bp}(28.15 \%)$, and the least frequent alleles were 453 and 458 bp $(0.42 \%)$. The most frequent genotypes occurring were 309/309, 309/357 and 309/445 bp, each with the proportion of $6.72 \%$. A total of 14 alleles and 27 genotypes were observed in Nicobari with allele size varying from 193 to $552 \mathrm{bp}$. In this breed the most and least frequent alleles were $343 \mathrm{bp}(50.4 \%)$ and 354, 357 and $552 \mathrm{bp}(0.40 \%)$, respectively. The most frequent genotypes were on the order of $343 / 343(29.6 \%), 261 / 343(19.2 \%)$ and 261/261 bp $(8.0 \%)$. In WLH, a total of 10 alleles were seen with the most and least frequent ones being $261 \mathrm{bp}(66 \%)$ and $565 \mathrm{bp}$ $(0.42 \%)$, respectively. The allele size varied from 241 to $565 \mathrm{bp}$, and among the 17 observed genotypes, 261/261 (47.9\%), 261/403 (9.2\%) and 261/552 bp (8.4\%) genotypes were the most frequently occurring ones.

Number of genotypes, number of observed alleles $\left(N_{\mathrm{a}}\right)$ and effective alleles $\left(N_{\mathrm{e}}\right)$ were highest in Ghagus followed by Nicobari and WLH breeds (Table 1). Similarly, the number of private alleles was highest in the Ghagus breed $(78.3 \%$ of total 23 alleles). The next-highest number of private alleles was observed in Nicobari (57.1\% of total 14 alleles) followed by the WLH (50\% of total 10 alleles) breed. Private alleles are those alleles which occurred only in a particular breed and not shared by other breeds, at least in this study.

Expected and unbiased expected heterozygosities were highest in Ghagus followed by Nicobari and WLH breeds (Table 2). As a result, significant $(P>0.0001)$ difference in heterozygosity among the three breeds was observed (Table 3). Heterozygosity was high in Ghagus and Nicobari breeds with a proportion of $84 \%$ and $57.6 \%$ heterozygous genotypes, respectively, as compared to $49.6 \%$ of heterozygous genotypes in the WLH breed. Based on observed and expected genotype frequencies, it was observed that the WLH breed did not deviate from Hardy-Weinberg Equilibrium (HWE) $(p=0.331)$, whereas Ghagus and Nicobari breeds significantly $(p<0.0001)$ deviated from HWE. The genetic distance (Nei) between Ghagus and Nicobari breeds was higher as compared to that of Ghagus and WLH and that between Nicobari and WLH breeds (Table 4).

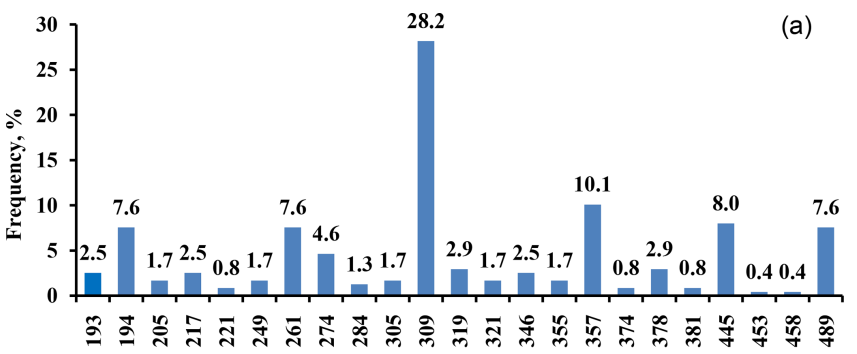

Alleles

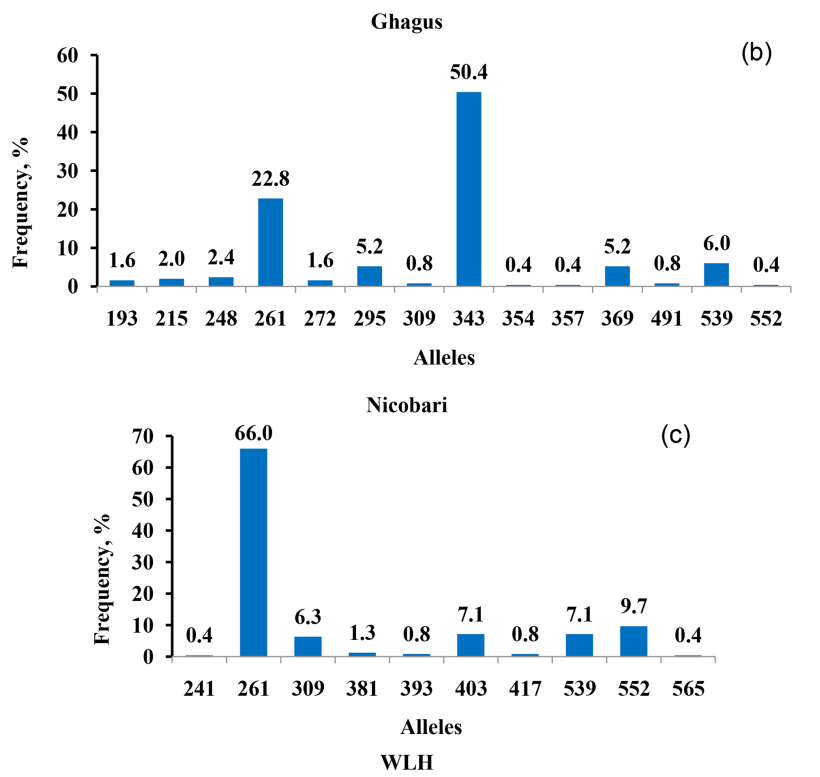

Figure 1. Allele frequency of LEI0258 locus in (a) Ghagus, (b) Nicobari and (c) WLH breeds.

Significant association was observed between alleles of the LEI0258 marker and body weight in the Ghagus breed (Table 5). As compared to the reference (309 bp) allele, the $194 \mathrm{bp}$ allele had a significant positive influence on body weight recorded at 4 and 8 weeks of age. The $194 \mathrm{bp}$ allele also had a significant positive influence on shank length at 8 weeks of age, egg production and survivability up to 72 weeks of age. However, the $445 \mathrm{bp}$ allele had a significant negative effect on body weight recorded at 40 weeks of age. This allele also had a nonsignificant negative influence on body weight recorded at other ages. None of the alleles had a significant effect on age at first egg, shank length at 16 weeks of age and immunity traits measured at 20 weeks of age in this breed.

In the Nicobari breed, out of 16 alleles observed, 261 and 295 bp alleles had a significant positive association with antibody titres to NDV vaccine at 20 weeks of age when compared to the reference allele (343 bp). However, no alleles were found to be significantly associated with growth, production, NAb titres to RRBC or survivability traits in this breed (Table 5). 
Table 2. Effective number of alleles, heterozygosity and fixation index in three chicken breeds.

\begin{tabular}{lrrrrrrr}
\hline Breeds & $N_{\mathrm{a}}$ & $N_{\mathrm{e}}$ & $N_{\mathrm{pa}}$ & $H_{\mathrm{o}}$ & $H_{\mathrm{e}}$ & $u H_{\mathrm{e}}$ & $F$ \\
\hline Ghagus & 23.0 & 8.3 & 18 & 0.83 & 0.88 & 0.88 & 0.05 \\
Nicobari & 14.0 & 3.2 & 8 & 0.58 & 0.68 & 0.69 & 0.16 \\
WLH & 10.0 & 2.2 & 5 & 0.53 & 0.54 & 0.54 & 0.02 \\
\hline Mean \pm SE & $15.7 \pm 3.80$ & $4.5 \pm 1.9$ & & $0.65 \pm 0.09$ & $0.70 \pm 0.09$ & $0.70 \pm 0.10$ & $0.08 \pm 0.04$ \\
\hline
\end{tabular}

$N_{\mathrm{a}}$ : observed number of alleles; $N_{\mathrm{e}}$ : effective number of alleles; $N_{\mathrm{pa}}$ : number of private alleles; $H_{\mathrm{O}}$ : observed heterozygosity; $H_{\mathrm{e}}:$ expected heterozygosity; $u H_{\mathrm{e}}$ : unbiased expected heterozygosity; $F$ : fixation index.

Table 3. Percentage of homozygous and heterozygous individuals among three breeds.

\begin{tabular}{|c|c|c|c|c|c|c|}
\hline \multirow{2}{*}{$\begin{array}{l}\text { Breed } \\
\text { Status }\end{array}$} & \multicolumn{2}{|c|}{ Ghagus } & \multicolumn{2}{|c|}{ Nicobari } & \multicolumn{2}{|c|}{ WLH } \\
\hline & No. & $\%$ & No. & $\%$ & No. & $\%$ \\
\hline Heterozygous & 100 & 84.03 & 72 & 57.60 & 59 & 49.58 \\
\hline Homozygous & 19 & 15.97 & 53 & 42.40 & 60 & 50.42 \\
\hline Total & 119 & 100 & 125 & 100 & 119 & 100 \\
\hline
\end{tabular}

$\chi$ value $33.58 ; P>0.0001$.

Table 4. Genetic identity/distance among three breeds of chicken.

\begin{tabular}{lrrr}
\hline Population/breed & Ghagus & Nicobari & WLH \\
\hline Ghagus & - & 0.106 & 0.293 \\
Nicobari & 2.244 & - & 0.411 \\
WLH & 1.227 & 0.889 & - \\
\hline
\end{tabular}

Genetic identity (above the diagonal) and genetic distance (below the diagonal) were calculated from allele frequency according to Nei's (1978) unbiased estimates.

In WLH, the 403 bp allele had a significant negative influence on body weight recorded at 16 weeks of age. The negative effect of this allele was also observed on body weight recorded at a different age, but they were not significant. When compared to the reference allele (261 bp), 309 and $552 \mathrm{bp}$ alleles were significantly negatively associated with the egg production up to 72 weeks of age. The $309 \mathrm{bp}$ allele was also significantly negatively associated with survivability of hens up to 72 weeks of age. No allele was found to have significant effect on shank length, age at first egg or immunity traits in this breed (Table 5).

\section{Discussion}

Considering the number of alleles, genotypes and heterozygosity of the LEI0258 VNTR locus, it was evident that genetic diversity at MHC in indigenous breeds was higher than that of the WLH breed. Indigenous breeds were not subjected to artificial selection, and only natural selection was operating in their rearing environment to favour individuals with higher resistance to various diseases that prevailed in the field as MHC is linked with disease resistance, and hence the higher diversity was observed in indigenous breeds. Furthermore, the Ghagus breed was recently collected from its native tract (Six generations back), while WLH was subjected to selection for higher egg production for last several generations, and hence, some degree of inbreeding could have taken place in this breed. Previous studies also reported higher diversity in the MHC-linked LEI0258 locus in indigenous chickens of various countries. A high number of alleles of LEI0258 was reported in eight ecotypes (46 alleles) of Kenya (Ngeno et al., 2014), three indigenous breeds (25 alleles) of Iran (Nikbakht et al., 2013) and in 33 indigenous breeds (69 alleles) of China (Han et al., 2013). On the other hand, lesser genetic variability and diversity at MHC were reported in intensively selected commercial egg layer varieties (Izadi et al., 2011) and commercial white egg layers (Chazara et al., 2013) previously. Higher allelic diversity observed in indigenous chicken breeds can act as a source of unique or novel MHC haplotype types that could be helpful in deciding breeding strategies for conservation and improvement of indigenous chickens for rearing in free-range or backyard systems of production.

The most frequently observed allele was $261 \mathrm{bp}$ among the three breeds. The alleles that were common to the three breeds were 261 and $309 \mathrm{bp}$. Finding of the high frequency of the $261 \mathrm{bp}$ allele in WLH $(66.0 \%)$ and to some extent in Nicobari $(22.8 \%)$ breeds as compared to the Ghagus $(7.6 \%)$ breed could be explained by the fact that the $261 \mathrm{bp}$ allele is known to be associated with the B15 haplotype (Fulton et al., 2006), and this haplotype is reported to be linked with the high egg production (Lunden et al., 1993). WLH is an eggtype improved breed which was selected for higher egg production, while Nicobari is known to have the highest egg production among indigenous breeds of India. Therefore high frequency of the $261 \mathrm{bp}$ allele was observed in these two breeds.

The present study uncovered 12 unique/novel alleles (248, $272,274,284,346,354355,374,378,403$ and $565 \mathrm{bp}$ ) that were not reported previously either by genotyping or by sequencing (Lima-Rosa et al., 2005; Fulton et al., 2006; Lwelamira et al., 2008; Schou et al., 2010; Chazara et al., 2013; Nikbakht et al., 2013; Han et al., 2013; Guangxin et al., 
Table 5. Association of LEI0258 alleles with survivability, production and antibody traits in indigenous and WLH breeds of chicken.

\begin{tabular}{lrrrr}
\hline Traits & LEI0258 allele, bp & Allele effect & SE & $P$ value \\
\hline Ghagus & & & & \\
\hline Body weight at 4 weeks & 194 (BW3) & 34.8 & 14.5 & 0.025 \\
Body weight at 8 weeks & 194 & 100.7 & 42.4 & 0.030 \\
Body weight at 16 weeks & 194 & 111.3 & 62.8 & NS \\
Shank length at 8 weeks & 194 & 7.59 & 2.33 & 0.003 \\
Egg production up to 72 weeks & 194 & 60.9 & 29.6 & 0.05 \\
Survivability up to 72 weeks & 194 & 93.7 & 39.3 & 0.048 \\
Body weight at 40 weeks & 445 & -263.2 & 86.4 & 0.003 \\
\hline Nicobari & & & & \\
\hline SpAb titre to NDV at 20 weeks of age & 261 (B15 or B2) & 0.99 & 0.43 & 0.023 \\
& $295(\mathrm{~B} 5)$ & 0.58 & 0.26 & 0.026 \\
\hline WLH & & & & \\
\hline Body weight at 16 weeks & 309 (B24) & -81.1 & 27.3 & 0.016 \\
Egg production up to 72 weeks & $552(\mathrm{~B} 19.1)$ & -48.7 & 19.3 & 0.013 \\
& 309 & -53.2 & 20.4 & 0.011 \\
\hline Survivability up to 72 weeks & & & & \\
\hline
\end{tabular}

Estimates of allele effects relative to $309 \mathrm{bp}$ (reference allele) in Ghagus, $343 \mathrm{bp}$ (reference allele) in Nicobari and $261 \mathrm{bp}$ (reference allele) in the WLH breed based on formula $Y_{i}=\mu+\Sigma b_{j} f_{i j}+\varepsilon_{i}$. SE: standard error; NS: not significant.

2014; Esmailnejad et al., 2017; Mwambene et al., 2019). The size of 403 and $565 \mathrm{bp}$ alleles were confirmed by sequencing. However, the size of remaining alleles was determined by extrapolating the genotyped sizes. Therefore, the precise size of these alleles needs to be confirmed by sequencing. Nevertheless, the findings suggest the possibility of indigenous chickens (seven in Ghagus, three in Nicobari and two in WLH) possessing the unique alleles of the LEI0258 marker. Only BW3 (194 bp), 13.1 (381 bp), 19 (539 bp) and 19.1 (552 bp) serologically defined B haplotypes could be correlated among the three breeds. These findings are in conformity with the previous study, which reported that birds of Indian origin had completely unknown haplotypes, and only few serologically defined haplotypes (B2, B12 and B21) could be detected in four lines of indigenous chickens of India (Baelmans et al., 2005), thus reflecting potentially interesting genetic pool in indigenous breeds.

Ghagus and Nicobari breeds deviated from HWE. The deviation from HWE occurs due to selection (natural or artificial), missing genotypes, genetic drift, mutation, migration, population substructure, genotyping error, etc. (Chen et al., 2017). Genotyping error in this study was ruled out as sizes of alleles were first determined on agarose gel electrophoresis, and the precise size of all alleles were then determined on automated analyser, and the procedures used were common to all three breeds. The possible reason for deviation could be missing genotypes, population substructure or natural selection observed in both indigenous breeds of chicken. Similar deviation from HWE was reported with high heterozygosity at MHC in chicken populations originated in China (Izadi et al., 2011). Similar to the findings of our study, Mwambene et al. (2019) also reported significant deviation from HWE for the LEI0258 marker in 6 out of 10 indigenous chicken ecotypes of Africa. It was opined that deficiency of heterozygosity (lesser $H_{\mathrm{o}}$ than $H_{\mathrm{e}}$ ) or excess heterozygosity could be one among many reasons for the deviation. In our case perhaps excess heterozygosity observed particularly in Ghagus could be one of the reasons for deviation from HWE. Expected and unbiased expected heterozygosities were similar in all three breeds. Fixation index $(F)$, which indicates degree of inbreeding was the lowest in Ghagus followed by WLH and Nicobari breeds. $F$ statistic (Fst) observed for this LEI0258 marker was 0.172 . This suggests that $17.2 \%$ of the genetic variation was due to breed differences, and the rest of the variation was due to differences between individuals within each breed, suggesting significant genetic differentiation among the three breeds (Wright, 1978).

The genetic distance (Nei) between Ghagus and Nicobari breeds was higher as compared to Ghagus and WLH and that between Nicobari and WLH breeds. Mainland India and Andaman and Nicobar islands are separated by Bay of Bengal, and therefore there was least chance for genetic relatedness among these two indigenous breeds. Closer genetic relatedness observed between Nicobari and WLH was somewhat unexpected, and this can be explained by the fact that the Nicobari breed was reported to have originated from the crosses of different exotic breeds such as WLH, Australorp, Plymouth Rock, etc., and indigenous fowl of the Nicobar group of islands (Chatterjee et al., 2005). Therefore, closer genetic relationship was observed between Nicobari 
and WLH breeds. Therefore, Ghagus was genetically distant from both Nicobari and WLH breeds. Similar to the findings of the present study, Ahlawat et al. (2004) reported the high genetic similarity (0.80) between Nicobari and WLH breeds of chicken using RAPD analysis.

Alleles of LEI0258 had a significant association with body weight, egg production and survivability traits in Ghagus and WLH breeds and antibody titres to NDV in the Nicobari breed. Significant positive association of LEI0258 alleles with body weight at 16 weeks of age was also reported in Tanzanian native chickens (Lwelamira et al., 2008), and negative association with body weight at 8 and 12 weeks of age was reported in Iranian Khorasan native chickens (Nikbakht and Esmailnejad, 2015). Survivability of hens is an important economic trait which has bearing on production performance. A positive association of $194 \mathrm{bp}$ allele with egg production up to 72 weeks of age in Ghagus can be explained from the fact that the survivability of hens with the $194 \mathrm{bp}$ allele was also significantly higher as compared to the reference allele (309 bp). Allele $309 \mathrm{bp}$, which had negative association with survivability of hens up to 72 weeks of age, also had negative association with egg production up to 72 weeks of age. This might be an indirect association as hens which survive for shorter/longer duration will have lesser/higher chances of producing less/more eggs. Egg production up to 72 weeks of age was highest in hens with the $261 \mathrm{bp}$ allele (reference alleles), which has the highest frequency in the WLH breed. Similarly, the survivability of hens having the $261 \mathrm{bp}$ allele was highest as compared to other alleles (except 403 bp allele) in the WLH breed. Significant association of LEI0258 alleles with the egg laying intensity trait was also reported in Khorasan indigenous chickens of Iran (Nikbakht and Esmailnejad, 2015). Another study reported the significant association of alleles of the BF2 locus of MHC with early and late mortality in commercial broiler line (Ewald et al., 2007) suggesting the role of MHC haplotypes in survivability trait of chickens. However, to our knowledge this is the first study to report the effects of LEI0258 alleles on duration of the survivability of hens during the 72-week laying period.

In Nicobari, LEI0258 alleles (261 and 295 bp) were positively associated with antibody titres to NDV vaccine. Similar to the findings of the present study, significant association of some of the LEI0258 alleles with antibody response to NDV was also reported in Tanzanian chicken ecotypes (Lwelamira et al., 2008) and broiler chickens (Esmailnejad et al., 2017). These findings reiterate the importance of MHC in vaccine response to the NDV antigen. Among viral diseases, incidence of Newcastle disease is the highest in native chickens reared in backyard or free-range systems of production. Therefore, information on diversity at MHC helps in devising breeding strategies for conservation and improvement of native chickens.

Associations between LEI0258 alleles and survivability and immune traits observed in the present study can result from direct effect of MHC genes, which are known to influence disease resistance and immune competence traits. Those of production traits may be due to indirect association between survivability (longer duration of production) and egg production up to 72 weeks of age. Almost all associations of LEI0258 alleles with production traits were found to be breed specific. This is due to the fact that (except for the $261 \mathrm{bp}$ allele) no alleles were found to be common to all three breeds and that reference alleles were different among the three breeds to determine the effect of each allele in comparison to the reference allele. Therefore, allelic effects on various traits need to be studied breed/line-wise (Ewald et al., 2007) and this is particularly relevant in indigenous chicken breeds due to prevalence of high diversity at MHC.

\section{Conclusions}

From the findings of the present study, it is concluded that the LEI0258 marker was highly polymorphic in indigenous breeds, and the number of alleles identified in each breed was different. Considering various parameters examined like number of observed and effective number of alleles, genotypes, heterozygosity, etc., it was evident that indigenous chicken breeds showed higher genetic variability at the MHC region over WLH egg-layer birds. Furthermore, the indigenous breeds likely to have novel alleles of the LEI0258 marker. Some of the alleles were found to have significant association with growth, production, survivability and antibody titres to NDV. Further studies are required to explore the MHC diversity in indigenous chickens through sequencing of the LEI0258 marker for SNP and indel polymorphisms and also to investigate the effects of novel alleles on various immune-competences, disease resistance and production traits using large number of samples particularly in indigenous chicken breeds.

Data availability. The data from the paper are available upon request from the corresponding author.

Author contributions. SH conceived and designed the study, analysed the data and drafted the paper. DD conducted the experiment KR, and RU was involved in drafting of the paper. RNC supported the study and critically reviewed the paper. All authors read and approved the final draft of the paper.

Competing interests. The authors declare that they have no conflict of interest.

Acknowledgements. The present study was funded by the Science and Engineering Research Board (SERB) of the Ministry of Science and Technology, govt. of India (SB/SO/AS-015/2014), as 
an Extramural Core Research Grant. The help of Appavoo Dhandapani of ICAR-NAARM, Hyderabad, and Gholamreza Nikbakht, Faculty of Veterinary Medicine, University of Tehran, Iran, in analysing the data is gratefully acknowledged.

Financial support. This research was supported by the Science and Engineering Research Board (grant no. SB/SO/AS-015/2014).

Review statement. This paper was edited by Steffen Maak and reviewed by two anonymous referees.

\section{References}

Ahlawat, S. P. S., Sunder, J., Kundu, A., Chatterjee, R. N., Rai, R. B., Kumar, B., Senani, S., Saha, S. K., and Yadav, S. P.: Use of RAPD-PCR for genetic analysis of Nicobari fowl of Andaman, Brit. Poultry Sci., 45, 194-200, 2004.

Baelmans, R., Parmentier, H. K., Nieuwland, M. G., Dorny, P., and Demey, F.: Serological screening for MHC (B)-polymorphism in indigenous chickens, Trop. Anim. Health Pro., 37, 93-102, 2005.

Banat, G. R., Tkalcic, S., Dzielawa, J. A., Jackwood, M. W., Saggese, M. D., Yates, L., Kopulos, R., Briles, W. E., and Collisson, E. W.: Association of the chicken MHC B haplotypes with resistance to avian coronavirus, Dev. Comp. Immunol., 39, 430 437, 2013

Besbes, B.: Genotype evaluation and breeding of poultry for performance under sub-optimal village conditions, World Poultry Sci. J., 65, 260-271, 2009.

Chatterjee, R. N. and Yadav, S. P.: Farming System of Nicobari Fowl - An Endangered Breed of Andaman and Nicobar Islands, India, World Poultry Sci. J., 4, 245-256, 2008.

Chatterjee, R. N., Yadav, S. P., and Rai, R. B.: Breed descriptor of Nicobari fowl. Bulletin, Central Agricultural Research Institute, Port Blair, Andaman and Nicobar Islands, India, 32 pp., 2005.

Chazara, O., Chang, C., Bruneau, N., Benabdeljelil, K., Fotsa, J., Kayang, B. B., Loukou, N. E., Osei-Amponsah, R., Yapi-Gnaore, V., Youssao, I. A. K., Chen, C., Pinard-van der Laan, M., TixierBoichard, M., and Bed'Hom, B.: Diversity and evolution of the highly polymorphic tandem repeat LEI0258 in the chicken MHC-B region, Immunogenetics, 65, 447-459, 2013.

Chen, B., Cole, J. W., and Grond-ginsbach, C.: Departure from Hardy Weinberg Equilibrium and genotyping error, Front. Genet., 8, 167, https://doi.org/10.3389/fgene.2017.00167, 2017.

Emam, M., Mehrabani-yeganeh, H., Barjesteh, N., Nikbakht, G., Thompson-crispi, K., Charkhkar, S., and Mallard, B.: The influence of genetic background versus commercial breeding programs on chicken immunocompetence, Poultry Sci., 93, 77-84, 2014

Esmailnejad, A., Brujeni, G. N., and Badavam, M.: LEI0258 microsatellite variability and its association with humoral and cell mediated immune responses in broiler chickens, Mol. Immunol., 90, 22-26, 2017.

Ewald, S. J., Ye, X., Avendano, S., McLeod, S., Lamont, S. J., and Dekkers, J. C. M.: Associations of BF2 alleles with antibody titres and production traits in commercial pure line broiler chickens, Anim. Genet., 38, 174-176, 2007.
Fulton, J. E., Juul-Madsen, H. R., Ashwell, C. M., McCarron, A. M., Arthur, J. A., O'Sullivan, N. P., and Taylor Jr., R. L.: Molecular genotype identification of the Gallus gallus major histocompatibility complex, Immunogenetics, 58, 407-421, 2006.

Guangxin, E., Sha, R., Zeng, S., Wang, C., Pan, J., and Han, J.: Genetic variability, evidence of potential recombinational event and selection of LEI0258 in chicken, Gene, 537, 126-131, 2014.

Han, B., Lian, L., Qu, L., Zheng J., and Yang, N.: Abundant polymorphisms at the microsatellite locus LEI0258 in indigenous chickens, Poultry Sci., 92, 3113-3119, 2013.

Haunshi, S., Burramshetty, A. K., Kannaki, T. R., Raja Ravindra, K. S., and Chatterjee, R. N.: Pattern recognition receptor genes expression profiling in indigenous chickens of India and White Leghorn, Poultry Sci., 96, 3052-3057, 2017.

Haunshi, S., Arun Kumar, B., Kannaki, T. R., and Rajkumar, U.: Survivability, immunity, growth and production traits in indigenous and White Leghorn breeds of chicken, Brit. Poultry Sci., 60, 683-690, 2019.

Izadi, F., Ritland, C., and Cheng, K. M.: Genetic diversity of the major histocompatibility complex region in commercial and noncommercial chicken flocks using the LEI0258 microsatellite marker, Poultry Sci., 90, 2711-2717, 2011.

Kannaki, T. R., Reddy, M. R., Dhanutha, N. R., Shanmugam, M., Chatterjee, R. N., Rajkumar, U., and Haunshi, S.: Differential expression of Toll-like receptor mRNA in White Leghorn and indigenous chicken of India, Vet. Res. Commun., 34, 633-639, 2010.

Karnati, H. K., Pasupuleti, S. R., Kandi, R., Undi, R. B., Sahu, I., Kannaki, T. R., Subbiah, M., and Gutti R. K.: TLR-4 signalling pathway: MyD88 independent pathway up-regulation in chicken breeds upon LPS treatment, Vet. Res. Commun., 39, 73-78, 2015.

Lima-Rosa, C. A. V., Canal, C. W., Fallavena, P. R. V., Freitas, L. B., and Salzano, F. M.: LEI0258 microsatellite variability and its relationship to B-F haplotypes in Brazilian (blue-egg Caipira) chickens, Genet. Mol. Biol., 28, 386-389, 2005.

Lunden, A., Edfors-Lilja, I., Johansson, K., Liljedahl, L. E., and Simonsen, M.: Associations between major histocompatibility complex genes and production traits in White Leghorns, Poultry Sci., 72, 989-999, 1993.

Lwelamira, J., Kifaro, G. C., Gwakisa, P. S., and Msoffe, P. L. M.: Association of LEI0258 microsatellite alleles with antibody response against Newcastle disease virus vaccine and body weight in two Tanzania chicken ecotypes, Afr. J. Biotech., 7, 714-720, 2008.

Mwambene, P. L., Kyallo, M., Machuka, E., Githae, D., and Pelle, R.: Genetic diversity of 10 indigenous chicken ecotypes from Southern Highlands of Tanzania based on Major Histocompatibility Complex-linked microsatellite LEI0258 marker typing, Poultry Sci., 98, 2734-2746, 2019.

McConnell, S. K., Dawson, D. A., Wardle, A., and Burke, T.: The isolation and mapping of 19 tetranucleotide microsatellite markers in the chicken, Anim. Genet., 30, 183-189, 1999.

Miller, M. M. and Taylor Jr., R. L.: Brief review of the chicken Major Histocompatibility Complex: the genes, their distribution on chromosome 16, and their contributions to disease resistance, Poultry Sci., 95, 375-392, 2016.

Nei, M.: Analysis of gene diversity in subdivided populations, $\mathrm{P}$. Natl. Acad. Sci. USA, 70, 3321-3323, 1973. 
Nei, M.: Estimation of average heterozygosity and genetic distance from a small number of individuals, Genetics, 76, 379-390, 1978.

Ngeno, K., Van Der Waaij, E. H., Megens, H. J., Kahi, A. K., Van Arendonk, J. A. M., and Crooijmans, R. P. M. A.: Genetic diversity of different indigenous chicken ecotypes using highly polymorphic MHC-linked and non-MHC microsatellite markers, Anim. Genet. Resources, 56, 1-7, https://doi.org/10.1017/S2078633614000484, 2014.

Nikbakht, G. and Esmailnejad, A.: Chicken major histocompatibility complex polymorphism and its association with production traits, Immunogenetics, 67, 247-252, 2015.

Nikbakht, G., Esmailnejad, A., and Barjesteh, N.: LEI0258 Microsatellite variability in Khorasan, Marandi, and Arian Chickens, Biochem. Genet., 51, 341-349, 2013.

Owen, J. P., Delany, M. E., and Mullens, B. A.: MHC haplotype involvement in avian resistance to an ectoparasite, Immunogenetics, 60, 621-631, 2008.

Peakall, R. and Smouse, P. E.: GenAlEx 6: Genetic analysis in Excel. Population genetic software for teaching and research, Mol. Ecol. Notes, 6, 288-295, 2006.

Peakall, R. and Smouse, P. E.: GenAlEx 6.5: Genetic analysis in Excel. Population genetic software for teaching and research an update, Bioinformatics, 28, 2537-2539, 2012.

Rai, R. B. and Ahlawat, S. P. S.: Evaluation of disease resistance characteristics of Nicobari fowl, Ind. Vet. J., 72, 354-357, 1995.

Sambrook, J. and Russell, D. W.: Molecular cloning: a laboratory manual, 3 Vol., Cold Spring Harbor Laboratory Press, New York, 2288 pp., 2001.
Schou, T. W., Labouriau, R., Permin, A., Christensen, J. P., Sørensen, P., Cu, H. P., Nguyen, V. K., and Juul-Madsen, H. R.: MHC haplotype and susceptibility to experimental infections (Salmonella Enteritidis, Pasteurella multocida or Ascaridia galli) in a commercial and an indigenous chicken breed, Vet. Immunol. Immunop., 135, 52-63, 2010.

Sunder, J., Chatterjee, R. N., Rai, R. B., Ahlawat, S.P.S., Kundu, A., Senani, S., Saha, S. K., Yadav, S. P., and Bhagat, D.: Outbreak of infectious bursal disease in poultry of A and $\mathrm{N}$ Islands, Ind. Vet. Med. J., 28, 23-25, 2004.

Suzuki, K. T., Matsumoto, E., Kobayashi, E., Matsumoto, T., Kobayashi, E., Uenishi, H., Churkina, I., Plastow, G., Yamashita, H., Hamasima, N., and Mitsuhashi, T.: Genotypes of chicken major histocompatibility complex B locus associated with regression of Rous sarcoma virus J-strain tumors, Poultry Sci., 89, 651657, 2010.

Wright, S.: Variability within and among natural populations, in: Evolution and the genetics of populations, Vol. 4, University of Chicago Press, Chicago, 590 pp., 1978.

Yadav, S. P., Kannaki, T. R., Mahapatra, R. K., Paswan, C., Bhattacharya, T. K., Sarkar, S. K., and Chatterjee, R. N.: In vivo cellmediated immune, hemagglutination inhibition response, hematological and biochemical values in native vs. exotic chicken breeds, Poultry Sci., 97, 3063-3071, 2018. 